\title{
Quantifying Bound and Active Antibodies Conjugated to Gold Nanoparticles: A Comprehensive and Robust Approach To Evaluate Immobilization Chemistry
}

\author{
Kiran Tripathi and Jeremy D. Driskell*(1) \\ Department of Chemistry, Illinois State University, Normal, Illinois 61790, United States
}

Supporting Information

\begin{abstract}
Gold nanoparticles (AuNPs) functionalized High Ab with antibodies have the potential to improve biosensing Loading Poor $\mathrm{Ab}$ $\approx \operatorname{Low} A b \times \operatorname{Good} A b$ technology because of the unique optical properties of AuNPs and the specificity of antibody-antigen interactions. Critical to the development and optimization of these AuNP-enabled sensing technologies is the immobilization of the antibody onto the AuNP. The development of novel immobilization strategies that optimize antibody loading and orientation in an effort to enhance antibody activity, and therefore assay performance, has been the focus of many recent studies. However, few analytical methods exist to accurately quantify the activity of conjugated antibodies and reliably compare different immobilization strategies. Herein, we describe an

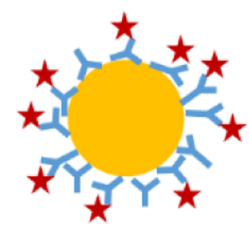

Nonspecific adsorption (non-oriented)

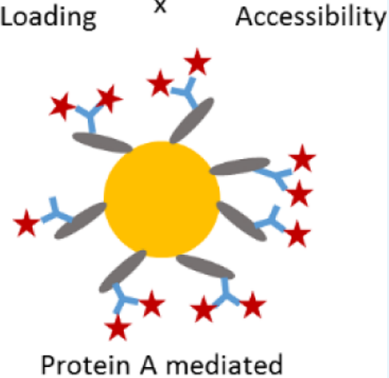

Protein A mediated (oriented) enzyme-mediated assay to quantify the fraction of the immobilized antibodies that is accessible for antigen binding. Antihorseradish peroxidase (anti-HRP) antibody is mixed with AuNPs to allow for conjugation, and the unbound, excess antibody is quantified with a modified Bradford assay to determine antibody loading onto AuNPs. The conjugates are then mixed with excess HRP to saturate all accessible binding sites, and bound HRP is quantified based on enzymatic reaction rate. This analytical scheme was used to compare two common immobilization strategies, nonspecific adsorption and protein A-mediated immobilization. We found that the antibody surface coverage is greater for direct adsorption than protein A-mediated binding; however, $23 \pm 6 \%$ of the directly adsorbed antibodies were active, whereas $91 \pm 19 \%$ of the antibodies bound through protein A were active. In addition to establishing this method as quantitatively precise and accurate, our results emphasize the need to quantify both antibody loading and antibody activity upon conjugation to gain greater insight into differences in immobilization chemistries and identify optimum protein conjugation strategies to maximize immunoassay performance.
\end{abstract}

\section{INTRODUCTION}

Antibody (Ab) immobilization onto gold nanoparticles (AuNPs) greatly affects $\mathrm{Ab}$-antigen binding and is critical to the success of AuNP-enabled immunoassays. It has been established that the antigen-binding capacity correlates with the number of accessible Fab domains. ${ }^{1-7}$ Thus, immobilized $\mathrm{Ab}$ surface coverage and orientation govern the analytical performance of these assays. Not surprisingly, then, several conjugation methods have been explored in search of an optimal immobilization protocol; ${ }^{8-14}$ however, analytical tools to accurately assess $\mathrm{Ab}$ loading and orientation are needed to compare different coupling chemistries on AuNPs.

Several instrumental methods have been developed to compare immobilization chemistries for planar surfaces. ${ }^{13}$ Atomic force microscopy, ${ }^{15,16}$ mass spectrometry (time-offlight secondary ion mass spectrometry), ${ }^{17,18}$ dual polarization interferometry, ${ }^{6,19}$ neutron reflectometry, ${ }^{19}$ spectroscopic ellipsometry, ${ }^{19-21}$ and total internal reflection ellipsometry $^{22-24}$ have all been used to assess $\mathrm{Ab}$ orientation, surface coverage, and binding capacity on planar surfaces. However, these methods are not suitable for the analysis of AuNPs, and relatively few approaches have been developed for the analysis of $\mathrm{Ab}$ immobilization on AuNPs. $\mathrm{Ab}$ immobilization chemistry onto AuNPs is often empirically evaluated by comparing the activity of the $\mathrm{Ab}-\mathrm{AuNP}$ conjugate in a functional assay. Conjugates formed using different immobilization chemistries have been incorporated into enzyme-linked immunosorbent assays, ${ }^{9,14}$ lateral flow assays, ${ }^{12}$ and aggregation-based assays, ${ }^{9,25}$ and improvements in the detection limit of those assays were attributed to proper orientation of the $A b$. However, this approach is limited in that it does not account for differences in $\mathrm{Ab}$ loading density and can result in an inappropriate interpretation. An increase in conjugate activity can result from proper $\mathrm{Ab}$ orientation or optimal loading density on the AuNP surface. Conversely, novel immobilization chemistries that lead to properly oriented immobilization may result in a decreased assay performance if coupling efficiency is poor. Thus, both the loading density and

Received: March 28, 2018

Accepted: July 12, 2018

Published: July 25, 2018 


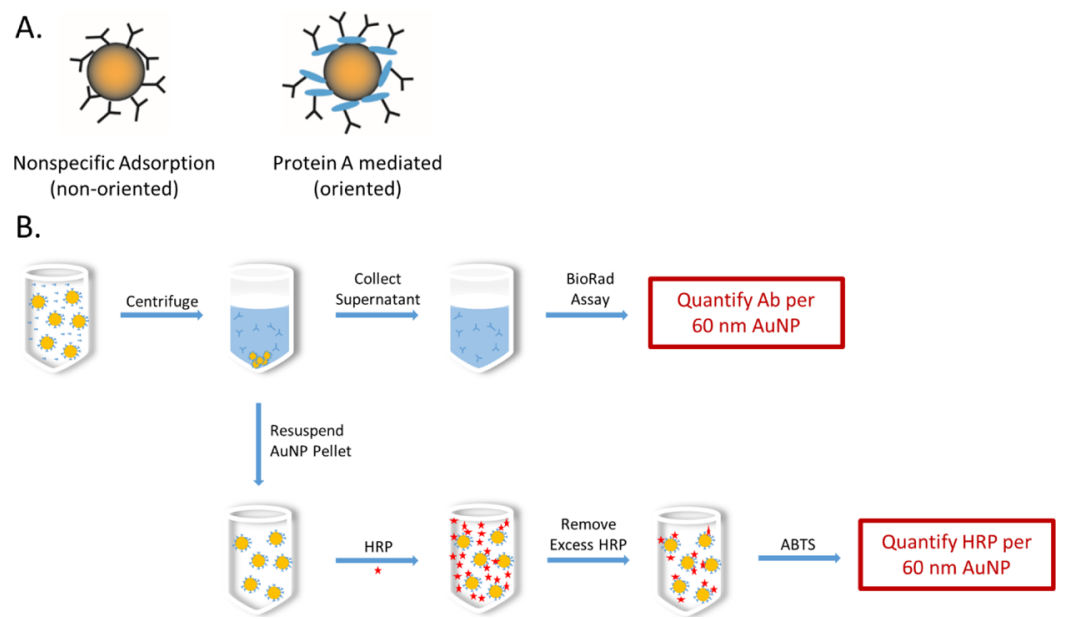

Figure 1. Immobilization strategies to prepare $\mathrm{Ab}-\mathrm{AuNP}$ conjugates which include nonspecifically adsorbed $\mathrm{Ab}$ for random orientation and $\mathrm{Ab}$ immobilization via protein A for controlled orientation (A). Illustration of the analytical approach to quantify both the number of Abs bound to each AuNP and the number of active binding sites presented by the immobilized Ab (B). Analysis of the supernatant containing the excess unbound $\mathrm{Ab}$ allows for quantitation of the number of adsorbed Abs on each AuNP. Active binding sites on the immobilized Ab are then saturated with HRP, and an enzymatic reaction enables quantitation of available binding sites presented by the immobilized Abs.

accessible binding sites must be measured quantitatively so that differences in $\mathrm{Ab}$ orientation can be evaluated and immobilization chemistries can be systematically compared.

Few studies have been reported that provide information on both the activity and accessibility of Abs immobilized on nanoparticles. Recently, Saha et al. utilized radioisotope $\left({ }^{125} \mathrm{I}\right)$ labeled Abs to accurately quantify the total Abs immobilized onto the AuNP, followed by ${ }^{125}$ I-labeled secondary $\mathrm{Ab}$ or protein $\mathrm{G}$, to gain insight into the accessibility of the Fab domain and $\mathrm{Ab}$ orientation. ${ }^{5}$ In this work, we immobilized anti-horseradish peroxidase (HRP) onto AuNPs and capitalized on the enzymatic activity of the antigen to quantify the fraction of immobilized Abs that is active toward antigens. This enzyme-mediated assay has been previously explored as a semiquantitative method to compare relative amounts of immobilized Abs. ${ }^{10}$ Anti-HRP and an enzymatic assay were used by Puertas et al. to evaluate $\mathrm{Ab}$ loading and antigen binding capacity on larger magnetic particles; ${ }^{12}$ however, the focus of that study was to evaluate the effects of immobilization conditions rather than the analytical method and precise quantitative information was not provided. In this report, we establish an enzyme-based analytical method that provides precision and sensitivity to quantitatively evaluate different immobilization chemistries on AuNPs. Moreover, we believe that this method can be easily implemented as a standardized approach to directly compare immobilization strategies.

\section{RESULTS AND DISCUSSION}

2.1. Overview. We have selected two common methods to immobilize Abs onto AuNPs in an effort to develop and apply a comprehensive analytical approach to compare immobilization methods (Figure 1A). First, Abs were directly adsorbed onto AuNPs. This strategy relies on hydrophobic, hydrophilic, and electrostatic interactions between the $\mathrm{Ab}$ and AuNP surface to form the conjugate. ${ }^{26-28}$ A more recent investigation also suggests that chemisorption through the cysteine thiol moiety is responsible for direct adsorption. ${ }^{29}$ Regardless of the interaction mechanism, this strategy does not control the orientation of the immobilized $\mathrm{Ab}$. In a second approach to form $\mathrm{Ab}-\mathrm{AuNP}$ conjugates, we utilized protein $\mathrm{A}$ to mediate the immobilization of the Ab onto AuNPs. This strategy results in appropriately oriented immobilization in which the antigenbinding sites of the $\mathrm{Ab}$ are directed toward the solution and are accessible for interacting with the antigen.

The general approach to quantify the surface concentration and orientation of the immobilized $\mathrm{Ab}$ is illustrated in Figure 1B. Anti-HRP is an ideal Ab in this analytical scheme because it provides a straightforward means to quantify accessible antigen-binding sites, that is, $\mathrm{Ab}$ orientation, by the enzymatic activity of the bound HRP antigen. Briefly, excess Ab is added to the AuNP suspension and allowed to saturate the AuNP surface. Excess unbound $\mathrm{Ab}$ is quantified using a modified Bradford assay, and the surface concentration of the immobilized Abs is calculated as the difference between the total $\mathrm{Ab}$ added and the excess $\mathrm{Ab}$ remaining in the supernatant. The $A b-A u N P$ conjugates are then reacted with excess HRP to saturate accessible binding sites on the immobilized Abs. The number of captured HRP is quantitatively correlated with the enzymatic activity via external calibration using standard solutions of HRP.

2.2. Characterization of Ab-AuNP Conjugates. UVvisible spectrophotometry and nanoparticle tracking analysis (NTA) were used to monitor the immobilization of Abs onto AuNPs. Unconjugated AuNPs exhibited an extinction maximum at $534 \mathrm{~nm}$ (Figure 2). A slight red shift in the extinction maximum to $538 \mathrm{~nm}$ was observed upon direct adsorption of anti-HRP Ab onto the AuNPs. This spectral shift resulted from a change in the local refractive index due to the protein adlayer and is consistent with previous reports. ${ }^{30}$ The hydrodynamic diameter also increased from $63.4 \pm 0.4$ to 75.9 $\pm 1.6 \mathrm{~nm}$ after direct adsorption of the Ab to the AuNP and is characteristic of a single IgG adlayer (Table S1). ${ }^{31-33}$ A second AuNP conjugate was formed by the chemisorption of thiolated protein A onto the AuNP followed by affinity coupling of anti$\mathrm{HRP} \mathrm{Ab}$. The extinction maxima for the protein A and protein $\mathrm{A} /$ anti-HRP $\mathrm{Ab}$ conjugates were located at 536 and $539 \mathrm{~nm}$, respectively, and the hydrodynamic diameters measured 85.5 \pm 1.3 and $95.5 \pm 1.2 \mathrm{~nm}$, respectively. It is worth noting that the thickness of the protein A layer is greater than that of the $\mathrm{Ab}$ layer. Protein $\mathrm{A}$ is a rodlike protein with a length of $\sim 25-$ 


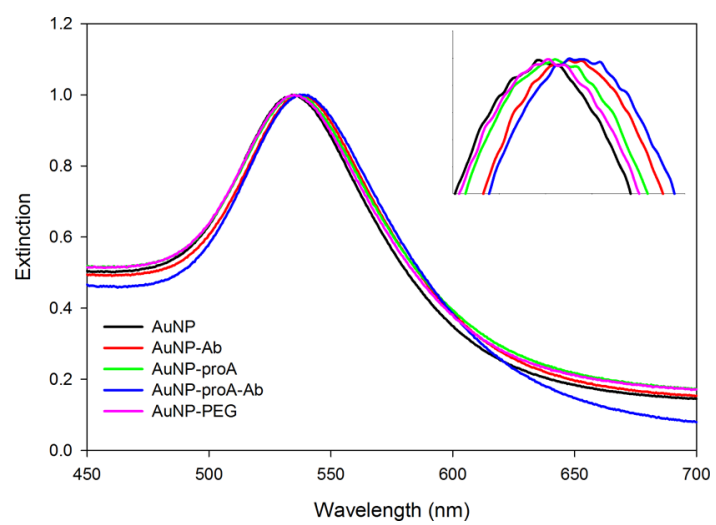

Figure 2. Characterization of the unconjugated and functionalized AuNPs by UV-visible spectrophotometry to monitor surface modification. Extinction spectra are provided for unconjugated AuNPs, Ab-modified AuNPs, protein A-modified AuNPs, protein Amodified AuNPs with a bound Ab, and PEG1000-modified AuNPs.

$30 \mathrm{~nm}$, which was previously shown to adsorb onto AuNPs at $26^{\circ}$ with respect to the surface normal for an overall increase in the hydrodynamic diameter of $22-24 \mathrm{~nm} .{ }^{32,33}$ Thus, the 22.1 $\mathrm{nm}$ increase in size for the protein A-modified AuNP relative to the unconjugated AuNP is consistent with these previously reported values. The hydrodynamic diameter increased $10.0 \pm$ $1.8 \mathrm{~nm}$ after the addition of the $\mathrm{Ab}$ to the protein A-modified AuNP and is similar to the $12.5 \pm 1.6 \mathrm{~nm}$ increase in size observed for direct adsorption of Abs onto AuNPs. A third AuNP sample was modified with thiolated PEG1000. Polyethylene glycol (PEG) modification of AuNPs has been shown to improve colloidal stability and minimize nonspecific protein binding; ${ }^{34}$ thus, PEG-AuNPs were prepared to serve as a negative control for antigen binding studies. PEG-functionalized AuNPs displayed an extinction maximum at $535 \mathrm{~nm}$ and a hydrodynamic diameter of $70.1 \pm 1.4 \mathrm{~nm}$. This observed increase in hydrodynamic diameter of $6.7 \mathrm{~nm}$ is consistent with a previous report measuring an increase of $7.2 \mathrm{~nm}$ for a PEG900 adlayer on AuNPs by dynamic light scattering. ${ }^{34}$ These red shifts in extinction maxima and increases in hydrodynamic diameters, relative to unconjugated AuNPs, confirmed surface modification of AuNPs.

2.3. Quantitation of the Abs Immobilized on AuNPs. As mentioned above, the $\mathrm{Ab}$ loading density is an essential parameter that must be measured quantitatively so that differences in $\mathrm{Ab}$ orientation can be evaluated and immobilization chemistries can be systematically compared. To this end, a modified Bradford assay was performed on the supernatant collected after a $1 \mathrm{~h}$ incubation of the anti-HRP $\mathrm{Ab}$ with unconjugated and protein A-modified AuNPs. Standard solutions of anti-HRP were used to generate a calibration curve to quantify the excess $\mathrm{Ab}$ in the supernatant (Figure $\mathrm{S} 1$ ). The number of $\mathrm{Ab}$ molecules adsorbed onto the AuNPs was calculated as the difference between the initial concentration of the $\mathrm{Ab}$ added to the AuNPs and the unbound $\mathrm{Ab}$ in the supernatant (Table S2). It should be noted that any loss of $\mathrm{Ab}$ due to adsorption onto the sample vial, for example, a microcentrifuge tube, would result in a systematic error that overestimates $\mathrm{Ab}$ loading onto the AuNP. To minimize this potential error, calibration standards were incubated in centrifuge tubes for the same time as the AuNP sample, and several low-binding microcentrifuge tubes were evaluated to select the product with minimal protein adsorption. A total of
24 independent AuNP samples, 12 conjugates formed via direct adsorption to AuNPs and 12 conjugates prepared with protein A-modified AuNPs, were analyzed. Direct adsorption of the $\mathrm{Ab}$ onto AuNPs resulted in a loading of $227 \pm 35 \mathrm{Abs} /$ AuNP. Theoretical monolayer coverage calculated based on the size of an $\mathrm{Ab}$ molecule would result in a surface density of $\sim 90 \mathrm{Ab} / \mathrm{AuNP}$ for side-on orientation and $2330 \mathrm{Ab} / \mathrm{AuNP}$ for end-on orientation. ${ }^{7,35,36}$ Thus, the measured value of 227 $\pm 35 \mathrm{Abs} / \mathrm{AuNP}$ falls within the expected range for monolayer coverage. To confirm full monolayer coverage and the absence of multilayers, the concentration of Abs added to the AuNP suspension was varied. Supernatant analysis as a function of added $\mathrm{Ab}$ confirms that $\mathrm{Ab}$ loading maximized at $\sim 225 \mathrm{Abs} /$ AuNP (Figure S2). The hydrodynamic diameter of the conjugates was also measured as a function of $\mathrm{Ab}$ concentration by NTA to evaluate the adsorbed Ab layer. Figure S3 demonstrates that the hydrodynamic diameter of the conjugates increased to a maximum of $\sim 71 \mathrm{~nm}$ and is consistent with a single layer of $\mathrm{Ab} .^{31,32}$

Ab loading onto protein A-modified AuNPs resulted in $68 \pm$ $10 \mathrm{Abs} / \mathrm{AuNP}$, which was significantly lower than that for direct adsorption. The decrease in Ab binding to protein Amodified AuNPs relative to direct adsorption onto AuNPs is likely the result of two factors. First, protein A is a rodlike protein with a long dimension of $25-30 \mathrm{~nm} .^{37}$ The size and shape of protein A leads to a larger effective footprint than IgG to result in fewer immobilized molecules on the AuNP; thus, there were fewer surface binding sites on the protein Amodified AuNP compared to an unmodified AuNP for Abs to attach. Second, protein A may not be properly oriented to bind the $\mathrm{Fc}_{\mathrm{c}}$ region of the $\mathrm{Ab}$, thus limiting access to $\mathrm{Ab}$ immobilization sites on the AuNP surface. This analysis highlights the variability in Ab loading among conjugation chemistries and underscores the need to quantify immobilized Abs when evaluating and comparing immobilization strategies.

2.4. Quantitation of Ab Binding Sites. Excess HRP was added to the $A b-A u N P$ conjugates to saturate all accessible antigen-binding sites for the immobilized Abs. On the basis of the maximum loading density of the $\mathrm{Ab}$ for the $\mathrm{Ab}-\mathrm{AuNP}$ conjugates prepared via direct adsorption, 30-fold excess HRP was added to ensure saturation. Moreover, equal quantities of HRP were captured by the Ab-AuNP conjugates for both $1 \mathrm{~h}$ and overnight $(\sim 18 \mathrm{~h})$ incubations to confirm that equilibrium was established for the $\mathrm{Ab}$-antigen interaction under these experimental conditions. As illustrated in Figure 1B, the captured HRP was quantified by an enzymatic reaction with 2,2'-azino-bis(3-ethylbenzothiazoline-6-sulfonic acid) (ABTS). This analytical approach assumes that the enzymatic activity of Ab-bound HRP captured on the conjugates is equivalent to that of the free HRP used for external calibration. To confirm that HRP enzymatic activity is not inhibited when bound by an $\mathrm{Ab}$, two sets of standard solutions were prepared, one consisting of free HRP and one consisting of HRP to which $\mathrm{Ab}-\mathrm{AuNP}$ conjugates were added. The HRP is complexed to the $\mathrm{Ab}$ for the standard solutions in which $\mathrm{Ab}-\mathrm{AuNP}$ conjugates were added. ABTS was added to the standard solutions, and the enzymatic reaction rate was determined from the absorbance of the formed product. The reaction rate was plotted as a function of HRP concentration to generate calibration curves (Figure 3), and the data established a linear correlation. Moreover, the linear calibration curves were equivalent for the free HRP and HRP captured by $\mathrm{Ab}-$ AuNP conjugates; thus, HRP captured by the Ab-AuNP 


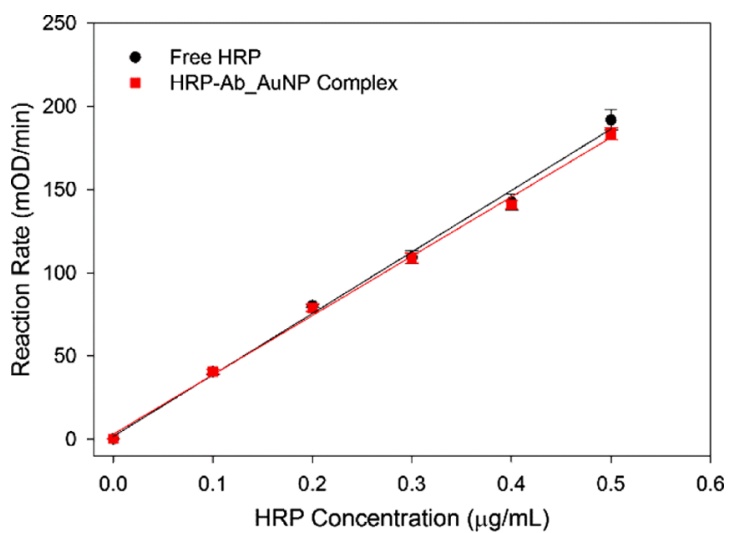

Figure 3. Calibration curves for HRP. The enzymatic reaction rate of HRP with ABTS is plotted as a function of HRP concentration. Calibration was prepared with standard solutions of free HRP and $\mathrm{HRP}$ bound to the $\mathrm{Ab}-\mathrm{AuNP}$ conjugates.

conjugates could be accurately quantified with external calibration.

Reaction rates for conjugates saturated with HRP are presented in Figure 4. Both of the $\mathrm{Ab}-\mathrm{AuNP}$ conjugates

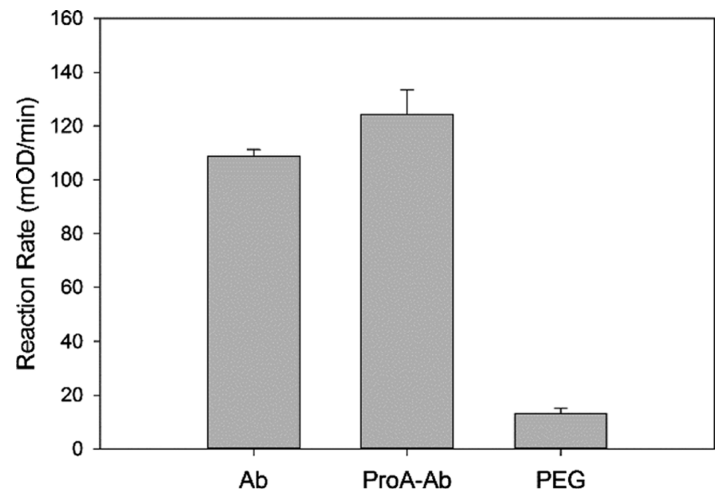

Figure 4. Quantitation of Ab-binding sites. AuNP conjugates were saturated with HRP and quantified based on the enzymatic reaction rate with ABTS. Three conjugates were prepared: direct adsorption of anti-HRP onto AuNPs, protein A-mediated immobilization of antiHRP onto AuNPs, and AuNPs modified with thiolated PEG1000 as a negative control.

resulted in a significant reaction rate, whereas the PEG-AuNP conjugate provided a minimal reaction rate. Signals for the $\mathrm{Ab}-\mathrm{AuNP}$ conjugates were corrected by subtracting the signal from that of the PEG-AuNP control conjugates that was due to the baseline reaction rate in the absence of enzyme and/or contribution of nonspecific HRP binding. Additional negative control conjugates were prepared with IgG to further confirm the binding specificity of the anti-HRP conjugate and validate the PEG-modified AuNP as an accurate measure of nonspecific binding. Figure S4 demonstrates that only anti-HRP conjugates captured HRP, whereas other IgG-modified AuNP conjugates demonstrated similar nonspecific adsorption of HRP as the PEG-AuNP conjugate. The quantity of Abcaptured HRP was calculated based on the calibration curve and normalized with respect to conjugate concentration to determine the number of captured HRP molecules per conjugate (Table 1). Extinction spectra were acquired to experimentally measure the AuNP conjugate concentration for each sample, which was critical because the AuNP concen-
Table 1. Summary of Fraction of Immobilized Abs That Maintains Antigen-Binding Activity ${ }^{a}$

$\begin{array}{lccc}\text { conjugate } & \mathrm{HRP} / \mathrm{AuNP} & \mathrm{Ab} / \mathrm{AuNP} & \% \text { active } \mathrm{Ab}(\%) \\ \mathrm{Ab} & 104 \pm 25 & 227 \pm 35 & 23 \pm 6 \\ \mathrm{ProA}-\mathrm{Ab} & 124 \pm 19 & 68 \pm 10 & 91 \pm 19\end{array}$

${ }^{a}$ Standard deviations are based on 12 independent preparations for each conjugate.

tration could vary by as much as $20 \%$ as a result of $6-9$ centrifugation/decant/resuspension cycles (3 cycles after protein $\mathrm{A}$ immobilization, 3 cycles after $\mathrm{Ab}$ immobilization, and 3 cycles after HRP binding). Interestingly, similar numbers of HRP molecules were captured by the conjugates formed via direct adsorption $(104 \pm 23 \mathrm{HRP} / \mathrm{AuNP})$ and protein Amediated immobilization $(124 \pm 17 \mathrm{HRP} / \mathrm{AuNP})$ given that significantly fewer Abs were loaded onto the protein A conjugates (Table 1).

The accuracy of this method to quantify accessible binding sites requires that all available binding sites are saturated with HRP and that all excess HRP molecules are removed from the conjugate suspension. To establish that these criteria were met, $\mathrm{Ab}-\mathrm{AuNP}$ conjugates were mixed with increasing amounts of HRP. The number of captured HRP molecules increased as the amount of added HRP increased and reached a maximum of 105 HRP/AuNP (Figure 5). These data confirm that all

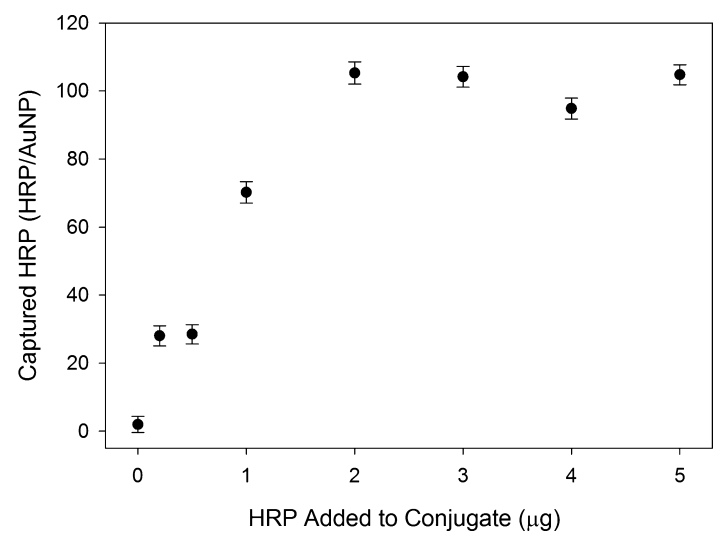

Figure 5. Quantitation of captured HRP molecules as a function of added HRP to determine the saturation of available binding sites presented by immobilized Abs.

available binding sites were saturated with HRP with the addition of $3 \mu \mathrm{g}$ of HRP used in previous experiments. A rinse study was performed to verify that three centrifugation/ resuspension rinse cycles were sufficient to remove all unbound HRP molecules from the conjugate suspensions. HRP $(3 \mu \mathrm{g})$ was added to the negative control PEG conjugates, and the number of centrifugation cycles to remove excess HRP from the conjugates was varied. Figure S5 shows that after two rinse cycles, nearly $98 \%$ of the HRP was removed from the negative control conjugates and no benefit was achieved with more than three rinse cycles, confirming that excess HRP was effectively removed from the conjugates to accurately quantify the bound HRP molecules.

The fraction of immobilized Abs with accessible antigenbinding sites was calculated by combining both the measured number of immobilized Abs per AuNP and captured HRP per AuNP (Table 1). Conjugates formed via direct adsorption loaded with $227 \pm 35 \mathrm{Abs} / \mathrm{AuNP}$ provide a maximum of 454 
antigen-binding sites per AuNP, taking into account the divalency of IgG Abs; however, each conjugate captured only $104 \pm 23$ HRP molecules. This suggests that $23 \pm 6 \%$ of the Ab-binding sites are properly oriented and accessible to interact with the antigen. This value is consistent with random orientation of the immobilized $\mathrm{Ab}$ and in agreement with a study by Caruso, et al., which found $27 \pm 7 \%$ of the binding sites occupied by antigens for randomly oriented Abs immobilized on smooth gold surfaces. ${ }^{38}$ Conversely, $68 \pm 10$ Abs loaded onto each protein A-modified AuNP result in a maximum of 136 antigen-binding sites per AuNP. Each protein A conjugate captured $124 \pm 17$ HRP molecules, suggesting that $91 \pm 19 \%$ of the Ab-binding sites are properly oriented and accessible to interact with the antigen. These results are consistent with previous work by Puertas et al. that found that properly oriented anti-HRP can bind two HRP molecules for $100 \%$ biding efficiency. ${ }^{12}$ Similarly, Tajima et al. demonstrated that protein A binds the $\mathrm{Fc}$ region of IgG to properly orient Abs, enabling the capture of $1.8 \pm 0.1$ antigen molecules per $\mathrm{Ab}$ ( $90 \pm 5 \%$ binding activity), provided that the antigen is smaller than $\sim 100 \mathrm{kDa} .{ }^{39}$ Collectively, these results validate this analytical approach as a comprehensive, robust, and accurate method to quantify the fraction of immobilized Abs that maintains antigen-binding activity and to compare immobilization strategies.

\section{CONCLUSIONS}

We have developed an accurate method that can be easily implemented as a standardized approach to directly compare immobilization strategies. We capitalized on the enzymatic activity of the target antigen to develop an analytical approach to quantify the accessibility of the active $\mathrm{Ab}$ conjugated to AuNPs. On the basis of previous work regarding $\mathrm{Ab}$ orientation, we hypothesized that $\mathrm{Ab}-\mathrm{AuNP}$ conjugates formed with protein $\mathrm{A}$ to facilitate $\mathrm{Ab}$ immobilization would result in a greater quantity of bound antigens and a larger fraction of accessible Abs when compared to conjugates formed through nonspecific $\mathrm{Ab}-\mathrm{AuNP}$ interactions. In support of our hypothesis, we found a statistically significant increase in antigen binding for the protein A-mediated conjugate, although this improvement in antigen binding was less significant than anticipated based on previous work. ${ }^{17,39}$ More notably, significantly more Abs were immobilized via nonspecific adsorption than via protein $\mathrm{A}$. Thus, taking into account both $\mathrm{Ab}$ loading and captured antigen, we found that $23 \pm 6 \%$ of nonspecifically adsorbed Abs remained accessible and active, whereas $91 \pm 19 \%$ of Abs immobilized via protein A were accessible, thereby supporting our hypothesis that protein A properly orients immobilized Abs to promote antigen binding. These results highlight the need to quantify both the $\mathrm{Ab}$ loading and activity to effectively evaluate immobilization chemistries.

\section{MATERIALS AND METHODS}

4.1. Reagents. AuNPs $(60 \mathrm{~nm})$ were purchased from Ted Pella Inc. (Redding, CA). HRP, borate buffer, and ABTS (1Step ABTS) were obtained from Thermo Scientific (Rockford, $\mathrm{IL}$ ). The mouse anti-HRP IgG monoclonal Ab (clone 2H11) was acquired from MyBioSource. Thiolated protein A was purchased from Protein Mods LLC (Madison, WI). Thiolmodified polyethylene glycol (SH-PEG 1000) was purchased from Creative PEGWorks (Durham, NC). Bio-Rad protein assay dye reagent concentrate was acquired from Bio-Rad Laboratories, Inc. (Hercules, CA). All aqueous solutions were prepared in NANO pure deionized water $(18 \mathrm{M} \Omega)$ from a Barnstead water purification system (Thermo Scientific, Rockford, IL).

4.2. Synthesis of Ab-AuNP Conjugates. 4.2.1. Direct Ab Immobilization (Nonoriented). AuNPs (60 nm; $100 \mu \mathrm{L}$ ) were placed into a low-binding microcentrifuge tube, and $4 \mu \mathrm{L}$ of $50 \mathrm{mM}$ borate buffer was added to adjust the $\mathrm{pH}$ of the suspension to 8.0. The mouse anti-HRP monoclonal $\mathrm{Ab}(4 \mu \mathrm{g})$ was added to the AuNP suspension and incubated for $1 \mathrm{~h}$ at 4 ${ }^{\circ} \mathrm{C}$ to allow for direct adsorption. After incubation, the functionalized AuNPs were centrifuged at $5000 \mathrm{~g}$ for $5 \mathrm{~min}$, the supernatant was decanted, and the pelleted nanoparticles were resuspended in $2 \mathrm{mM}$ borate buffer ( $\mathrm{pH} \mathrm{8.0)}$ ). The supernatant of the first centrifugation was collected for protein analysis to infer the quantity of the Abs immobilized on the AuNPs. The functionalized AuNPs were subsequently centrifuged/resuspended twice more to thoroughly remove excess Abs.

4.2.2. Protein A-Mediated Ab Immobilization (Oriented). The $\mathrm{pH}$ of AuNPs $(60 \mathrm{~nm})$ was adjusted to $\mathrm{pH} 8.0$ with the addition of $50 \mathrm{mM}$ borate buffer to a final concentration of 2 $\mathrm{mM}$. AuNPs were then incubated with $2 \mu \mathrm{g}$ of thiolated protein $\mathrm{A}$ for $1 \mathrm{~h}$ at $4{ }^{\circ} \mathrm{C}$. Excess protein $\mathrm{A}$ was removed by three centrifugation/washing cycles at $5000 \mathrm{~g}$ for $5 \mathrm{~min}$. During each wash, the supernatant was removed and the pelleted AuNPs were resuspended in $2 \mathrm{mM}$ borate buffer ( $\mathrm{pH} \mathrm{8.0)}$. To the protein A-modified AuNPs, $4 \mu \mathrm{g}$ of mouse anti-HRP $\mathrm{Ab}$ was added and allowed to incubate for $1 \mathrm{~h}$ at $4{ }^{\circ} \mathrm{C}$. The functionalized AuNPs were then centrifuged at $5000 \mathrm{~g}$ for 5 min and the pelleted AuNPs resuspended in $2 \mathrm{mM}$ borate buffer three times to remove the excess $A b$. The supernatant of the first wash was collected for the Ab quantitation.

4.2.3. PEG-Modified AuNPs. For control studies, AuNPs were modified with thiolated PEG1000. To this end, $15 \mu \mathrm{L}$ of $20 \mu \mathrm{M}$ thiol-PEG1000 was incubated with $100 \mu \mathrm{L}$ of $60 \mathrm{~nm}$ AuNPs and $4 \mu \mathrm{L}$ of $50 \mathrm{mM}$ borate buffer ( $\mathrm{pH} 8.0$ ) for $1 \mathrm{~h}$ at 4 ${ }^{\circ} \mathrm{C}$. Following incubation, the PEG-modified AuNPs were washed to remove excess PEG by three centrifugation/washing cycles at $5000 \mathrm{~g}$ for $5 \mathrm{~min}$.

4.3. Quantitation of Abs Conjugated to AuNPs. The number of Abs immobilized onto AuNPs was determined using a modified Bradford assay (Bio-Rad protein assay). Standard solutions of anti-HRP $\mathrm{Ab}(0-50 \mu \mathrm{g} / \mathrm{mL})$ were prepared in $2 \mathrm{mM}$ borate buffer $(\mathrm{pH}$ 8.0). Ab-AuNP conjugates and the standard solutions were centrifuged at $5000 \mathrm{~g}$ for $5 \mathrm{~min}$, and $90 \mu \mathrm{L}$ of the supernatant or standard solution was transferred to a 96-well plate. Each sample and standard was diluted with $70 \mu \mathrm{L}$ of $2 \mathrm{mM}$ borate buffer ( $\mathrm{pH}$ 8.0 ), followed by the addition of $40 \mu \mathrm{L}$ of the Bio-Rad reagent. The samples and standards were then incubated at room temperature for $10-15 \mathrm{~min}$ to allow the color to develop. UV-visible absorption was measured at $595 \mathrm{~nm}$ to quantify the Abs in the supernatant. For lower concentrations of Abs $(0-7 \mu \mathrm{g} / \mathrm{mL})$, the microassay protocol was required. The microassay calls for the addition of $200 \mu \mathrm{L}$ of Bio-Rad reagent to $800 \mu \mathrm{L}$ of sample or standard solutions, followed by an absorption measurement in a $1 \mathrm{~cm}$ sample cell. The total number of $\mathrm{Ab}$ molecules adsorbed onto the AuNPs was calculated as the difference between the number of $\mathrm{Ab}$ molecules added to the AuNP suspension and the number of Abs that remained in the supernatant. 
4.4. Quantitation of Ab Binding Sites. The AuNP conjugates were incubated with $3 \mu \mathrm{L}$ of $1 \mathrm{mg} / \mathrm{mL}$ HRP for $1 \mathrm{~h}$ to saturate all active $\mathrm{Ab}$ binding sites. The excess HRP was removed by three centrifugation/wash cycles at $5000 \mathrm{~g}$ for 5 min. A $10 \mu \mathrm{L}$ aliquot of the conjugate was mixed with $150 \mu \mathrm{L}$ of 1-Step ABTS substrate solution, and the enzymatic reaction rate was determined from the absorbance of the formed product at $415 \mathrm{~nm}$. Linear correlation between the concentration of HRP and the enzymatic reaction rate allows the quantification of the number of captured HRP on the AuNP conjugates via calibration with standard solutions of HRP.

4.5. Instrumentation. 4.5.1. UV-Visible Spectrophotometer. The extinction spectra of the unconjugated and surfacemodified AuNPs were acquired with a Cary 1 Bio UV-visible dual-beam spectrophotometer. The slit was set to $0.2 \mathrm{~nm}$, and measurements were collected at $0.5 \mathrm{~nm}$ increments over a range of 400-900 nm. A Bio-Rad, iMark Microplate Reader was used for high-throughput, simultaneous collection of absorbance data required for the Bio-Rad protein assay and the HRP-ABTS enzymatic reaction. To quantify the Abs in the AuNP supernatant via the Bio-Rad protein assay, the absorbance at $595 \mathrm{~nm}$ was measured. The iMark Microplate Reader was set to collect the absorbance at $415 \mathrm{~nm}$ at $10 \mathrm{~s}$ intervals for a total of $20 \mathrm{~min}$ to determine the HRP-ABTS enzymatic reaction rate.

4.5.2. Nanoparticle Tracking Analysis. Hydrodynamic diameters for the conjugates were measured with a NanoSight LM10 NTA system configured with an LM14 532 nm laser module and a high-sensitivity sCMOS camera. Samples were prepared at $\sim 10^{9}$ nanoparticles $/ \mathrm{mL}$, and the analysis was performed under a constant flow $(15 \mu \mathrm{L} / \mathrm{min})$ for improved sampling. The hydrodynamic diameter for each sample was based on the analysis of five $60 \mathrm{~s}$ videos, using a camera level of 7 and a detection threshold set to a value of 5. Using these video settings, approximately 10000 individual nanoparticles were analyzed for each sample to calculate a mean size and standard deviation.

\section{ASSOCIATED CONTENT}

\section{S Supporting Information}

The Supporting Information is available free of charge on the ACS Publications website at DOI: 10.1021/acsomega.8b00591.

Mean hydrodynamic diameters of conjugates, raw and processed data for Bio-Rad $\mathrm{Ab}$ assay, calibration curve for Bio-Rad $\mathrm{Ab}$ assay, saturation of AuNPs with Abs, mean hydrodynamic diameter of conjugates as a function of $\mathrm{Ab}$ concentration, evaluation of nonspecific binding of HRP to AuNP conjugates, and effect of rinsing cycles on removal of excess HRP (PDF)

\section{AUTHOR INFORMATION}

\section{Corresponding Author}

*E-mail: jdriske@ilstu.edu (J.D.D.).

ORCID $\odot$

Jeremy D. Driskell: 0000-0001-5082-898X

Notes

The authors declare no competing financial interest.

\section{ACKNOWLEDGMENTS}

This work was supported by the Defense Threat Reduction Agency, Basic Research Award no. HDTRA1-13-1-0028. Additional funding was provided by Illinois State University's Department of Chemistry and College of Arts and Sciences.

\section{REFERENCES}

(1) Joshi, P. P.; Yoon, S. J.; Hardin, W. G.; Emelianov, S.; Sokolov, K. V. Conjugation of Antibodies to Gold Nanorods through Fc Portion: Synthesis and Molecular Specific Imaging. Bioconjugate Chem. 2013, 24, 878-888.

(2) Kausaite-Minkstimiene, A.; Ramanaviciene, A.; Kirlyte, J.; Ramanavicius, A. Comparative Study of Random and Oriented Antibody Immobilization Techniques on the Binding Capacity of Immunosensor. Anal. Chem. 2010, 82, 6401-6408.

(3) Lin, P.-C.; Chen, S.-H.; Wang, K.-Y.; Chen, M.-L.; Adak, A. K.; Hwu, J.-R. R.; Chen, Y.-J.; Lin, C.-C. Fabrication of Oriented Antibody-Conjugated Magnetic Nanoprobes and Their Immunoaffinity Application. Anal. Chem. 2009, 81, 8774-8782.

(4) Puertas, S.; Moros, M.; Fernández-Pacheco, R.; Ibarra, M. R.; Grazú, V.; de la Fuente, J. M. Designing novel nano-immunoassays: antibody orientation versus sensitivity. J. Phys. D: Appl. Phys. 2010, 43, 474012

(5) Saha, B.; Songe, P.; Evers, T. H.; Prins, M. W. J. The influence of covalent immobilization conditions on antibody accessibility on nanoparticles. Analyst 2017, 142, 4247-4256.

(6) Song, H. Y.; Zhou, X.; Hobley, J.; Su, X. Comparative Study of Random and Oriented Antibody Immobilization as Measured by Dual Polarization Interferometry and Surface Plasmon Resonance Spectroscopy. Langmuir 2012, 28, 997-1004.

(7) Saha, B.; Evers, T. H.; Prins, M. W. J. How Antibody Surface Coverage on Nanoparticles Determines the Activity and Kinetics of Antigen Capturing for Biosensing. Anal. Chem. 2014, 86, 8158-8166.

(8) Montenegro, J.-M.; Grazu, V.; Sukhanova, A.; Agarwal, S.; de la Fuente, J. M.; Nabiev, I.; Greiner, A.; Parak, W. J. Controlled antibody/(bio-) conjugation of inorganic nanoparticles for targeted delivery. Adv. Drug Delivery Rev. 2013, 65, 677-688.

(9) Mustafaoglu, N.; Kiziltepe, T.; Bilgicer, B. Site-specific conjugation of an antibody on a gold nanoparticle surface for onestep diagnosis of prostate specific antigen with dynamic light scattering. Nanoscale 2017, 9, 8684-8694.

(10) Parolo, C.; de la Escosura-Muñiz, A.; Polo, E.; Grazú, V.; de la Fuente, J. M.; Merkoçi, A. Design, Preparation, and Evaluation of a Fixed-Orientation Antibody/Gold-Nanoparticle Conjugate as an Immunosensing Label. ACS Appl. Mater. Interfaces 2013, 5, 1075310759.

(11) Pei, Z.; Anderson, H.; Myrskog, A.; Dunér, G.; Ingemarsson, B.; Aastrup, T. Optimizing immobilization on two-dimensional carboxyl surface: $\mathrm{pH}$ dependence of antibody orientation and antigen binding capacity. Anal. Biochem. 2010, 398, 161-168.

(12) Puertas, S.; Batalla, P.; Moros, M.; Polo, E.; del Pino, P.; Guisán, J. M.; Grazú, V.; de la Fuente, J. M. Taking Advantage of Unspecific Interactions to Produce Highly Active Magnetic Nanoparticle-Antibody Conjugates. ACS Nano 2011, 5, 4521-4528.

(13) Trilling, A. K.; Beekwilder, J.; Zuilhof, H. Antibody orientation on biosensor surfaces: a minireview. Analyst 2013, 138, 1619-1627.

(14) van der Heide, S.; Russell, D. A. Optimisation of immuno-gold nanoparticle complexes for antigen detection. J. Colloid Interface Sci. 2016, 471, 127-135.

(15) Bergkvist, M.; Carlsson, J.; Oscarsson, S. A method for studying protein orientation with atomic force microscopy using relative protein volumes. J. Phys. Chem. B 2001, 105, 2062-2069.

(16) Iijima, M.; Somiya, M.; Yoshimoto, N.; Niimi, T.; Kuroda, S. Nano-visualization of oriented-immobilized IgGs on immunosensors by high-speed atomic force microscopy. Sci. Rep. 2012, 2, 790.

(17) Cho, I.-H.; Park, J.-W.; Lee, T. G.; Lee, H.; Paek, S.-H. Biophysical characterization of the molecular orientation of an 
antibody-immobilized layer using secondary ion mass spectrometry. Analyst 2011, 136, 1412-1419.

(18) Wang, H.; Castner, D. G.; Ratner, B. D.; Jiang, S. Probing the orientation of surface-immobilized immunoglobulin $\mathrm{G}$ by time-offlight secondary ion mass spectrometry. Langmuir 2004, 20, 18771887.

(19) Zhao, X.; Pan, F.; Cowsill, B.; Lu, J. R.; Garcia-Gancedo, L.; Flewitt, A. J.; Ashley, G. M.; Luo, J. Interfacial Immobilization of Monoclonal Antibody and Detection of Human Prostate-Specific Antigen. Langmuir 2011, 27, 7654-7662.

(20) Bae, Y. M.; Oh, B.-K.; Lee, W.; Lee, W. H.; Choi, J.-W. Study on orientation of immunoglobulin $\mathrm{G}$ on protein $\mathrm{G}$ layer. Biosens. Bioelectron. 2005, 21, 103-110.

(21) Xu, H.; Lu, J. R.; Williams, D. E. Effect of surface packing density of interfacially adsorbed monoclonal antibody on the binding of hormonal antigen human chorionic gonadotrophin. J. Phys. Chem. B 2006, 110, 1907-1914.

(22) Balevicius, Z.; Ramanaviciene, A.; Baleviciute, I.; Makaraviciute, A.; Mikoliunaite, L.; Ramanavicius, A. Evaluation of intact- and fragmented-antibody based immunosensors by total internal reflection ellipsometry. Sens. Actuators, B 2011, 160, 555-562.

(23) Baleviciute, I.; Balevicius, Z.; Makaraviciute, A.; Ramanaviciene, A.; Ramanavicius, A. Study of antibody/antigen binding kinetics by total internal reflection ellipsometry. Biosens. Bioelectron. 2013, 39, $170-176$.

(24) Makaraviciute, A.; Ramanavicius, A.; Ramanaviciene, A. Development of a reusable protein G based SPR immunosensor for direct human growth hormone detection in real samples. Anal. Methods 2015, 7, 9875-9884.

(25) Yuan, X.; Fabregat, D.; Yoshimoto, K.; Nagasaki, Y. Development of a high-performance immunolatex based on "soft landing” antibody immobilization mechanism. Colloids Surf., B 2012, $99,45-52$.

(26) Geoghegan, W. D.; Ackerman, G. A. Adsorption of horseradish peroxidase, ovomucoid and anti-immunoglobulin to colloidal gold for the indirect detection of concanavalin $\mathrm{A}$, wheat germ agglutinin and goat anti-human immunoglobulin $\mathrm{G}$ on cell surfaces at the electron microscopic level: a new method, theory and application. J. Histochem. Cytochem. 1977, 25, 1187-1200.

(27) Meissner, J.; Prause, A.; Bharti, B.; Findenegg, G. H. Characterization of protein adsorption onto silica nanoparticles: influence of $\mathrm{pH}$ and ionic strength. Colloid Polym. Sci. 2015, 293, 3381-3391.

(28) Verkleij, A. J.; Leunissen, J. L. Immuno-Gold-Labeling in Cell Biology; CRC Press, 1989.

(29) Wang, A.; Vangala, K.; Vo, T.; Zhang, D.; Fitzkee, N. C. A Three-Step Model for Protein-Gold Nanoparticle Adsorption. J. Phys. Chem. C 2014, 118, 8134-8142.

(30) Pollitt, M. J.; Buckton, G.; Piper, R.; Brocchini, S. Measuring antibody coatings on gold nanoparticles by optical spectroscopy. RSC Adv. 2015, 5, 24521-24527.

(31) Filbrun, S. L.; Driskell, J. D. A fluorescence-based method to directly quantify antibodies immobilized on gold nanoparticles. Analyst 2016, 141, 3851-3857.

(32) James, A. E.; Driskell, J. D. Monitoring gold nanoparticle conjugation and analysis of biomolecular binding with nanoparticle tracking analysis (NTA) and dynamic light scattering (DLS). Analyst 2013, 138, 1212-1218.

(33) Jans, H.; Liu, X.; Austin, L.; Maes, G.; Huo, Q. Dynamic Light Scattering as a Powerful Tool for Gold Nanoparticle Bioconjugation and Biomolecular Binding Studies. Anal. Chem. 2009, 81, 9425-9432.

(34) Liu, Y.; Shipton, M. K.; Ryan, J.; Kaufman, E. D.; Franzen, S.; Feldheim, D. L. Synthesis, Stability, and Cellular Internalization of Gold Nanoparticles Containing Mixed Peptide-Poly(ethylene glycol) Monolayers. Anal. Chem. 2007, 79, 2221-2229.

(35) Buijs, J.; Lichtenbelt, J. W. T.; Norde, W.; Lyklema, J. Adsorption of monoclonal IgGs and their $\mathrm{F}\left(\mathrm{ab}^{\prime}\right) 2$ fragments onto polymeric surfaces. Colloids Surf., B 1995, 5, 11-23.
(36) Sarma, V. R.; Silverton, E. W.; Davies, D. R.; Terry, W. D. The three-dimensional structure at 6 A resolution of a human gamma Gl immunoglobulin molecule. J. Biol. Chem. 1971, 246, 3753-3759.

(37) Ohnishi, S.; Murata, M.; Hato, M. Correlation between surface morphology and surface forces of protein A adsorbed on mica. Biophys. J. 1998, 74, 455-465.

(38) Caruso, F.; Rodda, E.; Furlong, D. N. Orientational aspects of antibody immobilization and immunological activity on quartz crystal microbalance electrodes. J. Colloid Interface Sci. 1996, 178, 104-115.

(39) Tajima, N.; Takai, M.; Ishihara, K. Significance of Antibody Orientation Unraveled: Well-Oriented Antibodies Recorded High Binding Affinity. Anal. Chem. 2011, 83, 1969-1976. 